\title{
BMJ Open Clinical effectiveness of manipulation and mobilisation interventions for the treatment of non-specific neck pain: protocol for a systematic review and meta-analysis
}

Elizabeth Bailey, ${ }^{1}$ Nicola R Heneghan (10 , ${ }^{2}$ Natasha J Cassidy, ${ }^{3}$ Deborah Falla (1) , ${ }^{2}$ Alison B Rushton (1D) 2,4

To cite: Bailey E, Heneghan NR, Cassidy NJ, et al. Clinical effectiveness of manipulation and mobilisation interventions for the treatment of nonspecific neck pain: protocol for a systematic review and meta-analysis. BMJ Open 2020;10:e037783. doi:10.1136/ bmjopen-2020-037783

- Prepublication history for this paper is available online. To view these files, please visit the journal online (http://dx.doi org/10.1136/bmjopen-2020037783).

Received 15 February 2020

Revised 28 June 2020

Accepted 10 July 2020
Check for updates

(C) Author(s) (or their employer(s)) 2020. Re-use permitted under CC BY-NC. No commercial re-use. See rights and permissions. Published by BMJ.

For numbered affiliations see end of article.

Correspondence to Professor Alison B Rushton; arushto3@uwo.ca

\section{ABSTRACT}

Introduction Non-specific neck pain (NSNP) is a common musculoskeletal condition resulting in pain, physical limitations and associated functional disability. Current guidelines recommend manipulation and/or mobilisation as part of the multimodal management of NSNP. This study focuses on intervention at the articular level and aims to identify whether joint mobilisation or joint manipulation has a greater effect on function, range of movement or pain outcomes in the management of NSNP.

Methods and analysis A systematic review protocol has been designed and is reported in line with the Preferred Reporting Items for Systematic Reviews and Meta-Analysis Protocols. A targeted search strategy will enable searching of key databases from inception to 31 March 2020: CINAHL, PEDro, AMED, EMBASE, OVID, MEDLINE, Web of Science, PubMed and Google Scholar. Key journals will be searched using predefined keywords determined from preliminary scoping searches for randomised controlled trials of manipulation and mobilisation modalities for adults with NSNP in the absence of radiculopathy or whiplash, published in English. Grey literature and unpublished studies will also be searched. Studies will be screened by title and abstract and full text. Two independent reviewers will conduct the searches independently, extract data, assess risk of bias (Cochrane Risk of Bias Tool 2) and assess overall strength of evidence (Grading of Recommendations, Assessment, Development and Evaluation). Meta-analysis will be performed where individual studies measure comparable outcomes including performance-based outcome measures such as range of movement or patient reported outcome measures such as Neck Disability Index; and where interventions are comparable in their delivery such as number of oscillations and Maitland grading. Where not possible, data will be presented descriptively.

Ethics and dissemination This study does not require ethical approval. Findings will be submitted for publication to relevant peer-reviewed journals and will be presented at profession-specific conferences. PROSPERO registration number CRD42020164457.
Strengths and limitations of this study

- This systematic review protocol has been designed in line with the Preferred Reporting Items for Systematic Reviews and Meta-Analysis Protocols and is registered on PROSPERO.

- The Cochrane Risk of Bias Tool 2 will be used to assess risk of bias of included trials by two reviewers.

- Grading of Recommendations, Development and Evaluation will be used to assess overall strength of evidence.

- By isolating the individual component that is, mobilisation and manipulation techniques from the multimodal treatment of non-specific neck pain, it is hoped that this review may reveal which technique yields the greatest change in outcomes.

- A potential limitation is that heterogeneity of interventions and outcomes may limit possible meta-analyses.

\section{INTRODUCTION}

\section{Rationale}

Neck pain is one of the biggest contributors to musculoskeletal disability, with a prevalence ranging between $16 \%$ and $75 \%$ worldwide, ${ }^{1}$ impacting on the physical, social and psychological well-being of an individual, as well as imparting domestic and socioeconomic implications on society and business. ${ }^{2}$ The global incidence of non-specific neck pain (NSNP) in the adult population is $4.9 \%$, with the highest incidence in North America (mean 6.5\%) followed by Western Europe (mean $6.3 \%$ ). ${ }^{3}$ Neck pain is the fourth greatest cause of global disability ${ }^{4}$ and affects between $30 \%$ and $50 \%$ of adults in any given year. ${ }^{5}$ Given the prevalence of neck pain, it is likely that most adults will experience neck pain at some point in their life. 
NSNP is defined as pain or discomfort in and around the neck and shoulder girdle, with or without pain or sensory changes into the arms,${ }^{6}$ with or without the loss of cervical range of movement $(\mathrm{ROM})^{7}$ in the absence of infection, inflammatory or structural pathology (eg, fracture).$^{8}$ Although the specific aetiology of NSNP is not known, it is considered largely multifactorial in nature, with poor posture, occupational activity, sporting activities, depression and mechanical injuries such as strains being cited. ${ }^{9}$ Based on the work of Kjaer et al, ${ }^{10}$ current clinical guidelines for $\mathrm{NSNP}^{11}$ recommend a multimodal approach to management including stretching, ROM exercises and manual therapy.

It is generally accepted that a multimodal approach is the most effective way of managing NSNP to include exercise, postural advice, manual therapy and acupuncture to name a few. There is little evidence about the efficacy of individual manual therapy interventions, and comparative clinical effectiveness in reducing pain and disability. Manual therapy interventions are used for people with NSNP with the aim of providing pain relief and restoring cervical ROM. ${ }^{12}$ Commonly used techniques directed at the articular structures are mobilisations of varying amplitude and manipulation. Mobilisations are passive accessory oscillations applied to the vertebral joints along the plane of movement. ${ }^{13}$ Manipulation has been defined as a high velocity, low amplitude, thrust manoeuvre applied in a perpendicular direction to the intended joint in order to engender movement at that joint, resulting in cavitation and surface separation of articular surfaces, ${ }^{14}$ however there are studies which describe manipulation is other combinations of movement and direction of thrust. ${ }^{15}$ The theory of surface separation has been demonstrated by computational vector analysis of forces software,$^{15}$ however there remains no evidence to show the real-time effects of joint separation, postulated to occur during manipulation.

Recent systematic reviews have identified that manipulation or mobilisation coupled with exercise is effective for the management of NSNP, but to date there is little evidence on the comparable effects of manipulation versus mobilisation. ${ }^{16}$ Existing evidence of manual therapy compares manipulation to other inert modalities such as placebo and sham, ${ }^{17}$ but few studies directly compare manual therapy modalities without additional interventions such as exercise. Current recommendations for manual therapy include thoracic and cervical manipulation or mobilisation, ${ }^{10}$ however there is no consensus on which is most efficacious in treating NSNP. This information would be valuable to informing best multimodal intervention packages. This study therefore aims to identify whether joint mobilisation or joint manipulation has a greater effect on function, range of motion or pain outcomes in the management of NSNP.

\section{Objectives}

1. To evaluate the effectiveness of manipulation intervention for NSNP on function, ROM and pain outcome measures.

2. To evaluate the effectiveness of mobilisation intervention for NSNP on function, ROM and pain outcome measures.

3. To evaluate whether there is a difference in outcome between manipulation and mobilisation interventions for NSNP on function, ROM and pain outcome measures.

\section{METHODS \\ Design}

A systematic review and meta-analysis will be conducted in accordance to this predefined protocol that is reported according to the Preferred Reporting Items for Systematic Reviews and Meta Analyses Protocols (PRISMA-P) checklist. The systematic review will be reported in line with the PRISMA statement and flow diagram. ${ }^{18}$

\section{Eligibility criteria}

Studies selected for inclusion will meet the following PICOS criteria. ${ }^{18}$ Studies not written in English will be excluded.

\section{Population}

Inclusion criteria: adults ( $>18$ years) experiencing NSNP of any duration of symptoms, with or without loss of range of movement. ${ }^{12}$

Exclusion criteria: radiculopathy, whiplash, inflammatory arthropathy, history of cervical surgery, myelopathy or other red flag symptoms, interventions in addition to manipulation or mobilisation.

\section{Intervention}

Objective 1: eligible studies will describe manipulative techniques of the thoracic or cervical spine, where manipulation is specifically a high velocity, low amplitude manoeuvre directed at the spine, classified as grade $\mathrm{V}$ or a thrust technique.

Objective 2: eligible studies will describe mobilisation techniques that involve a localised passive force delivered to the joint at a specific vertebral level, classified as grades I-IV depending on their amplitude within the joint's normal physiological ROM.

\section{Comparator}

Comparator studies can include placebo, sham or inactive controls.

\section{Outcome measures}

Any continuous or dichotomous patient-reported or performance-based outcome measure evaluating function, range of movement and pain. Eligible outcome measures include, but are not limited to: Neck Disability Index (NDI); Neck Pain Questionnaire; quality of life scores such as SF-36, Neck Pain and Disability Scale or 
Visual Analogue Scale pain score (or equivalent numeric rating scale) or ROM, which are validated, reliable and sensitive to change. Outcome measures will not be limited due to preliminary scoping searches showing few studies within the eligibility criteria.

\section{Study design}

Randomised controlled trials (RCTs), controlled clinical trials and cross-over trials will be eligible. Pilot and feasibility studies will not be included, where a feasibility study is defined as a preliminary study used to ascertain the best method by which a future study should take place, and a pilot study is defined as a small-scale study to test a research protocol, its data collection methods, sample, data handling tools, population and recruitment strategies in preparation for a larger scale study. ${ }^{19}$

Studies that directly compare manipulation or mobilisation will be included. Studies including other arms to trials such as acupuncture or exercise will be included where data are available per treatment arm. Studies with multiple arms will be considered if the interventions are in conjunction with the same additional modality, for example mobilisation plus exercise versus manipulation plus exercise, as long as the additional components are the same in terms of type and frequency.

\section{Information sources}

The search will use a sensitive subject and topic-based strategy from inception to 31 March 2020. Databases searched will be CINAHL, PEDro, AMED, EMBASE, OVID, MEDLINE, Web of Science, PubMed and Google Scholar. Manual searches of key journals for relevant articles, articles in press or published ahead of print will include Musculoskeletal Science \& Practice, European Spine Journal, Journal of Orthopaedic \& Sports Physical Therapy, Journal of Manual \& Manipulative Physiotherapy, and Spine. Grey literature will be searched in Zetoc, Open Grey and British National Bibliography.

\section{Search strategy}

Searches will be performed independently by primary and secondary reviewers (EB and NJC, respectively), both practising musculoskeletal physiotherapists. Databases will be searched using keywords and combinations of predefined keywords and search strings, tailored to each database. An example search strategy is shown for MEDLINE in box 1. Keywords will be grouped together by category (outcome measure, dysfunction, design, intervention) using boolean operators AND/OR/NOT and combined across categories to increase the sensitivity of the search (box 1). Modifications of keywords will take place to account for alternative spellings and synonyms used interchangeably (ie, manipulation and "high velocity thrust" or HVT); keywords are detailed in table 1.

\section{Study records}

Data management

Records of selected studies will be managed in Microsoft Word for Mac (V.2018) and EndNote V.X9 (2018).
Box 1 Example of simple search of keywords in MEDLINE including search term combinations at lines $15,28,33,47$ and 48

Database: Ovid MEDLINE(R)<1946 to June Week 2 2020>

Search strategy:

1. neck pain.mp. or Neck Pain/ (10870)

2. cervicodynia.mp. (9)

3. cervical pain.mp. (850)

4. cervicalgia.mp. (113)

5. neck strain.mp. (43)

6. neck ache.mp. (26)

7. arthralgia.mp. or Arthralgia/ (13035)

8. myalgia.mp. or Myalgia/ (7409)

9. spondylosis.mp. or Spondylosis/ or Cervical Vertebrae/ (37321)

10. neck injury.mp. or Neck Injuries/ (5362)

11. neck dysfunction.mp. (81)

12. cervical spine.mp. (19137)

13. stiffness.mp. (54668)

14. neck disorder.mp. (31)

15. 1 or 2 or 3 or 4 or 5 or 6 or 7 or 8 or 9 or 10 or 11 or 12 or 13 or 14 (127655)

16. neck disability index.mp. (1519)

17. neck disability questionnaire.mp. (3)

18. neck outcome score.mp. (4)

19. short form 36.mp. (8906)

20. outcome measure.mp. (54940)

21. disability.mp. (202611)

22. range of motion.mp. or "Range of Motion, Articular"/ (62641)

23. range of movement.mp. (2477)

24. VAS.mp. (41624)

25. Pain/ or Pain Measurement/ or visual analogue scale.mp. (205951)

26. NRS.mp. (5594)

27. Pain Measurement/ or numeric rating scale.mp. (86855)

28. 16 or 17 or 18 or 19 or 20 or 21 or 22 or 23 or 24 or 25 or 26 or 27 (529584)

29. controlled trial.mp. (533701)

30. crossover trial.mp. (5877)

31. clinical trial.mp. or Clinical Trial/ (683050)

32. randomi?ed controlled trial.mp. (525600)

33. 29 or 30 or 31 or 32 (937435)

34. Grade V.mp. (1210)

35. placebo.mp. (194046)

36. sham.mp. (75066)

37. manipulation.mp. or Manipulation, Orthopedic/ or Manipulation, Chiropractic/ or Manipulation, Spinal/ or Manipulation, Osteopathic/ (80610)

38. Thrust.mp. (3055)

39. high velocity.mp. (2698)

40. Manipulation, Orthopedic/ or Maitland.mp. or Physical Therapy Modalities/ or Musculoskeletal Manipulations/ (41727)

41. Musculoskeletal Manipulations/ or Mulligan.mp. (1883)

42. Physical Therapy Modalities/ or Physiotherapy.mp. (45827)

43. Osteopathic Medicine/ or osteopathy.mp. or Manipulation, Osteopathic/ (5172)

44. Manipulation, Chiropractic/ or Chiropractic/ or Chiropractic.mp. (5085)

45. adjustment.mp. (165339)

46. HVLA.mp. (77)

47. 34 or 35 or 36 or 37 or 38 or 39 or 40 or 41 or 42 or 43 or 44 or 45 or 46 (565851)

48. 15 and 28 and 33 and 47 (1284) 


\begin{tabular}{|c|c|c|c|}
\hline $\begin{array}{l}\text { Outcome } \\
\text { measure }\end{array}$ & Dysfunction & Design & Intervention \\
\hline $\begin{array}{l}\text { Neck disability } \\
\text { index }\end{array}$ & Cervical pain & Controlled trial & Adjustment \\
\hline $\begin{array}{l}\text { Neck pain } \\
\text { questionnaire }\end{array}$ & Cervicalgia & Crossover trial & Chiropractic \\
\hline $\begin{array}{l}\text { Neck outcome } \\
\text { score }\end{array}$ & Cervical spine & Clinical trial & Grade V \\
\hline Short form 36 & Cervicodynia & $\begin{array}{l}\text { Randomi?ed } \\
\text { controlled trial }\end{array}$ & High velocity \\
\hline $\begin{array}{l}\text { Outcome } \\
\text { measure }\end{array}$ & Neck strain & & HVLA \\
\hline Disability & Neck & & Maitland \\
\hline $\begin{array}{l}\text { Range of } \\
\text { motion }\end{array}$ & Neck pain & & Manipulation \\
\hline $\begin{array}{l}\text { Range of } \\
\text { movement }\end{array}$ & Neckache & & $\begin{array}{l}\text { Manual } \\
\text { therapy }\end{array}$ \\
\hline VAS & Arthralgia & & Mobili?ation \\
\hline $\begin{array}{l}\text { Visual analogue } \\
\text { scale }\end{array}$ & Myalgia & & Mulligan \\
\hline NRS & Spondylosis & & Osteopathy \\
\hline \multirow{4}{*}{$\begin{array}{l}\text { Numeric rating } \\
\text { scale }\end{array}$} & Neck injury & & Physiotherapy \\
\hline & $\begin{array}{l}\text { Neck } \\
\text { dysfunction }\end{array}$ & & Placebo \\
\hline & Neck disorder & & Sham \\
\hline & Stiffness & & Thrust \\
\hline
\end{tabular}

? denotes wildcard operator to account for variability in spelling, for example 'mobilisation' and 'mobilization'.

\section{Selection process}

Study selection will take place in two phases, (1) title and abstract and (2) full text, and will be performed by each reviewer independently against the inclusion and exclusion criteria. Titles and abstracts will be used to screen for suitability using a decision parameter of suitable/unsuitable/potentially eligible. Studies will be considered potentially eligible when on reviewing the abstract, it cannot be conclusively excluded. Full texts will be reviewed if suitability cannot be determined by the title and abstract alone, or in the case of disagreement. In the event of disagreement following discussion, a third reviewer (ABR) will have presiding decision. The study selection process will be reported in a PRISMA flow chart. $^{18}$

Cohen's $\kappa^{20}$ will be used to assess the level of agreement between reviewers at both stages of the review process. For this review, Cohen's original description of significance follows where $\kappa \leq 0.2$ will be considered no agreement, $0.21-0.59$ is weak, $0.6-0.79$ is moderate and over 0.8 is considered strong agreement.

\section{Data collection process}

Data will be extracted independently by EB and NJC. Data will be extracted using the Cochrane 'data
Table 2 Table of data items extracted from included studies

\begin{tabular}{ll}
\hline Content & Data items \\
\hline $\begin{array}{l}\text { General study } \\
\text { information }\end{array}$ & Author, year of publication, journal \\
Study characteristics & $\begin{array}{l}\text { Sample size, study design, } \\
\text { randomisation description }\end{array}$ \\
Participant Information & $\begin{array}{l}\text { Age, gender, chronicity of } \\
\text { symptoms }\end{array}$ \\
$\begin{array}{l}\text { Performance-based } \\
\text { outcome measure }\end{array}$ & $\begin{array}{l}\text { eg, Cervical range of motion, } \\
\text { muscle power }\end{array}$ \\
$\begin{array}{l}\text { Patient-reported } \\
\text { outcome measure }\end{array}$ & $\begin{array}{l}\text { eg, Neck Disability Index, Neck } \\
\text { Pain Questionnaire }\end{array}$ \\
Results & $\begin{array}{l}\text { Mean and SD of outcome } \\
\text { measures }\end{array}$ \\
\hline
\end{tabular}

collection forms for intervention reviews: RCTs and nonRCTs' tool. ${ }^{21}$ The tool will be initially piloted using five randomly selected studies and edited where necessary. The Template for Intervention Description and Replication (TIDieR) checklist will be used to describe the interventions reported in each included study. ${ }^{22}$ Interventions will be tabulated and pooled by intervention type.

\section{Data items}

Data to be extracted from included studies are summarised in table 2. Where data are missing from studies, authors will be contacted for clarification and completeness. Where possible, data regarding adverse events will be extracted; although from the preliminary scoping searches, this is anticipated to be minimal.

\section{Risk of bias in individual studies}

Risk of bias (ROB) will be assessed using the Cochrane Risk of Bias V.2 (CROB2) tool ${ }^{23}$ for each included study by the reviewers (EB, NJC). CROB2 has been selected as it is suitable to any type of randomised trial, and is considered comprehensive enough to be sensitive to all ROB, including selective reporting bias and bias arising from missing data; which other ROB tools are less sensitive to. ${ }^{24}$ ROB will be tabulated following an initial pilot on the same five randomly selected studies for piloting data extraction. The CROB2 tool considers random sequence generation, allocation concealment, blinding of participants and personnel, blinding of outcome assessment, incomplete outcome data, selective reporting and other biases, and will be used for each outcome measure in and across studies. ROB will be determined as high, uncertain or low. ${ }^{25}$

\section{Data synthesis}

The findings of the included studies will be synthesised and tabulated separately for manipulation techniques (objective 1) and mobilisation techniques (objective 2) by study characteristics, results and ROB. Details of interventions will be tabulated using selected components of the TIDier checklist. ${ }^{24}$ TIDier ensures completeness 
in the reporting of interventions and ensures detailed descriptions of the interventions under review. ${ }^{22}$

Meta-analysis will take place when outcomes and/ or interventions are heterogeneically low $\left(\mathrm{I}^{2}<50 \%\right)$. A random effects model will be used if there is clinical homogeneity, similarities between individuals, interventions and outcomes, ${ }^{26}$ and the statistical heterogeneity, the assessment of whether genuine differences exist between results ${ }^{27}$ is low. Interventions will be assessed for heterogeneity between studies with respect to modality, grading (ie, Maitland I-V), frequency and dosage. This will be determined from the recommendations from the Cochrane Handbook for Systematic Reviews of Interventions using $\mathrm{I}^{2}$, where $0 \%-40 \%$ is low, $30 \%-60 \%$ is moderate $50 \%-90 \%$ is substantial and $75 \%-100 \%$ is considerable heterogeneity. ${ }^{28}$ Continuous data will be quantitatively synthesised using standardised differences of mean $(95 \% \mathrm{CI}) \cdot{ }^{29}$ Dichotomous data will be analysed with risk ratios with $95 \%$ CI. ${ }^{30}$ Based on preliminary literature searches, it is anticipated that outcomes retrieved will allow for meta-analysis based on the following characteristics:

- Study design.

- Patient-reported outcome measure (specifically NDI).

- Performance-based outcome measure (specifically cervical ROM).

- Chronicity.

- Intervention type.

Where meta-analysis is not possible, a narrative synthesis will be presented, describing the types or study, variations within interventions, study design, outcome measures, comparability and comments about the study's overall quality. Groups of studies which are deemed to be heterogeneous will undergo narrative synthesis for interventions and comparator and outcome measured. Where narrative synthesis is undertaken, descriptions of variations within interventions, study design, outcome measures, populations, overall comparability and comments about the study's overall quality will be reported as well as extraneous variable which might affect the overall outcome. ${ }^{31}$

\section{Meta-biases}

To address potential reporting and publication biases, grey literature and conference proceedings will be searched to identify unpublished studies. Studies will be reviewed for reporting bias to ensure that all planned outcomes identified in the protocol for reporting were actually reported; they will be presented narratively and assessed against their protocol, where available, to ensure that all data have been reported. Results will be tabulated, describing the study design, sample, intervention, outcome measure and will be commented on regarding their quality.

\section{Confidence in cumulative evidence}

The strength of the overall body of evidence will be assessed using GRADE (Grading of Recommendations, Assessment, Development and Evaluation) to provide a transparent and systematic method of presenting evidence. $^{32}$ This approach includes the domains of ROB, imprecision (95\% CI around the best estimate of absolute effect), inconsistency (applicability of intervention outcomes in a population of interest) and publication bias (consideration of missing or excluded data) ${ }^{33}$ GRADE considers all factors which will contribute to the quality of the results, the evidence for the outcomes and the magnitude of the effect size. It ensures clarity in presenting the results and a systematic process to data review. The GRADE domains will be assessed to yield an arbitrary score of very low, low, medium or high and can be used to directly evaluate the methodological quality of studies included $;{ }^{34}$ and will demonstrate that the quality of evidence retrieved reflects the extent to which there is confidence that the point estimate is correct. GRADE will be used to determine the overall quality of evidence, and will be determined not serious (no change in quality) serious and very serious, which will in turn downgrade the quality to evidence levels 1 and 2 , respectively with no change for not serious ROB. ${ }^{21}$

\section{DISCUSSION}

Although current guidelines ${ }^{11}$ advocate a multimodal approach including manipulation and mobilisation for managing NSNP, there is a paucity of evidence of what specific intervention of manual therapy is most beneficial. By isolating the individual components within the multimodal treatment of NSNP, it is hoped that this review may reveal which manual therapy technique yields the greatest change in pain, function and disability outcomes. If effectiveness of each intervention is clear, this will inform selection of individual components of a multimodal intervention. Furthermore, there is no evidence of how best to deliver manual therapy in terms of frequency and dosage to optimise treatment outcomes in order to bring about a more expedient and complete resolution of symptoms and disability. Although this is not a primary focus of this review, preliminary data to inform a future study may be obtained. Furthermore, the added complexity of using mobilisations as a therapeutic intervention is that techniques are often delivered as a series of repeated movements within the patients' normal physiological range of motion classified as grades I-IV. Variability within 'dosage' delivered and grade of intervention may influence outcomes.

This review intends to analyse the difference, if any, between manipulation and mobilisations and intends to inform our understanding of what type of manual therapy may be most effective within the multimodal management of NSNP. Due to the incidence of NSNP in the general population and the cost burden to the economy, it is hoped that this review may inform best practice in implementing patient care and inform future comparator studies and clinical trials of manual therapy in order to deliver efficient, effective patient care.

Author affiliations

${ }^{1}$ Physiotherapy, University Hospitals Plymouth NHS Trust, Plymouth, UK 
${ }^{2}$ Centre of Precision Rehabilitation for Spinal Pain, School of Sport, Exercise and Rehabilitation Sciences, University of Birmingham, Birmingham, UK

${ }^{3}$ Physiotherapy, The Royal Orthopaedic Hospital, Birmingham, UK

${ }^{4}$ School of Physical Therapy, Western University, London, Ontario, Canada

Twitter Nicola R Heneghan @HeneghanNicola, Deborah Falla @Deb_Falla and Alison B Rushton @abrushton

Contributors All authors contributed towards the submitted final manuscript. EB is an MRes student at the University of Birmingham and a practising physiotherapist. $A B R$ is the lead supervisor, third reviewer and corresponding author. NRH and DF are co-supervisors. EB drafted the initial manuscript and received guidance on content, methodology and analysis from all supervisors. NJC was the second reviewer and is a physiotherapist. All authors have read and agreed the final manuscript.

Funding The authors have not declared a specific grant for this research from any funding agency in the public, commercial or not-for-profit sectors.

\section{Competing interests None declared.}

Patient and public involvement Patients and/or the public were not involved in the design, or conduct, or reporting, or dissemination plans of this research.

Patient consent for publication Not required.

Provenance and peer review Not commissioned; externally peer reviewed.

Open access This is an open access article distributed in accordance with the Creative Commons Attribution Non Commercial (CC BY-NC 4.0) license, which permits others to distribute, remix, adapt, build upon this work non-commercially, and license their derivative works on different terms, provided the original work is properly cited, appropriate credit is given, any changes made indicated, and the use is non-commercial. See: http://creativecommons.org/licenses/by-nc/4.0/.

ORCID iDs

Nicola R Heneghan http://orcid.org/0000-0001-7599-3674

Deborah Falla http://orcid.org/0000-0003-1689-6190

Alison B Rushton http://orcid.org/0000-0001-8114-7669

\section{REFERENCES}

1 Fejer R, Kyvik KO, Hartvigsen J. The prevalence of neck pain in the world population: a systematic critical review of the literature. Eur Spine J 2006;15:834-48.

2 Genebra CVDS, Maciel NM, Bento TPF, et al. Prevalence and factors associated with neck pain: a population-based study. Braz J Phys Ther 2017;21:274-80.

3 Hoy DG, Smith E, Cross M, et al. Reflecting on the global burden of musculoskeletal conditions: lessons learnt from the global burden of disease 2010 study and the next steps forward. Ann Rheum Dis 2015;74:4-7.

4 Cohen SP. Epidemiology, diagnosis, and treatment of neck pain. Mayo Clin Proc 2015;90:284-99.

5 Carroll LJ, Hogg-Johnson S, van der Velde G, et al. Course and prognostic factors for neck pain in the general population: results of the bone and joint decade 2000-2010 Task force on neck pain and its associated disorders. J Manipulative Physiol Ther 2009;32:S87-96.

6 Binder Al. Cervical spondylosis and neck pain. BMJ 2007;334:527-31.

7 Ganesh GS, Mohanty P, Pattnaik M, et al. Effectiveness of mobilization therapy and exercises in mechanical neck pain. Physiother Theory Pract 2015;31:99-106.

8 Hidalgo B, Hall T, Bossert J, et al. The efficacy of manual therapy and exercise for treating non-specific neck pain: a systematic review. $J$ Back Musculoskelet Rehabil 2017;30:1149-69.

9 Heintz MM, Hegedus EJ. Multimodal management of mechanical neck pain using a treatment based classification system. J Man Manip Ther 2008;16:217-24.
10 Kjaer P, Kongsted A, Hartvigsen J, et al. National clinical guidelines for non-surgical treatment of patients with recent onset neck pain or cervical radiculopathy. Eur Spine J 2017;26:2242-57.

11 National Institute for Health \& Care Excellence. Clinical knowledge summary: non-specific neck pain [online], 2018. Available: https:// cks.nice.org.uk/neck-pain-non-specific [Accessed Aug 2019].

12 Gross A, Langevin P, Burnie SJ, et al. Manipulation and mobilisation for neck pain contrasted against an inactive control or another active treatment. Cochrane Database Syst Rev 2015:CD004249.

13 Hengeveld E, Banks K. Maitland's vertebral manipulation. In: Management of neuromusculoskeletal disorders. 8th edn, 2013.

14 Evans DW, Lucas N. What is 'manipulation'? A reappraisal. Man Ther 2010;15:286-91.

15 Harwich AS. Joint manipulation: toward a general theory of highvelocity, low-amplitude Thrust techniques. J Chiropr Humanit 2017;24:15-23.

16 Blanpied PR, Gross AR, Elliott JM, et al. Neck pain: revision 2017: clinical practice guidelines linked to the International classification of functioning, disability and health from the orthopaedic section of the American physical therapy association. J Orthop Sports Phy Therapy 2017;47:A1-83.

17 Rubinstein SM, Terwee CB, Assendelft WJJ, et al. Spinal manipulative therapy for acute low-back pain. Cochrane Database Syst Rev 2012:CD008880.

18 Moher D, Liberati A, Tetzlaff J, et al. Preferred reporting items for systematic reviews and meta-analyses: the PRISMA statement. PLoS Med 2009;6:e1000097.

19 Hassan ZA, Schattner P, Mazza D. Doing a pilot study: why is it essential? Malays Fam Physician 2006;1:70-3.

$20 \mathrm{McHugh}$ ML. Interrater reliability: the kappa statistic. Biochem Med 2012;22:276-82.

21 Cochrane Training. Cochrane training [online], 2014. Available: http:// training.cochrane.org/resource/data-collection-forms-interventionreviews [Accessed Apr 2019].

22 Hoffmann TC, Glasziou PP, Boutron I, et al. Better reporting of interventions: template for intervention description and replication (TIDieR) checklist and guide. BMJ 2014;348:g1687.

23 Sterne JAC, Savović J, Page MJ, et al. RoB 2: a revised tool for assessing risk of bias in randomised trials. BMJ 2019;366:14898.

24 Page MJ, McKenzie JE, Higgins JPT. Tools for assessing risk of reporting biases in studies and syntheses of studies: a systematic review. BMJ Open 2018;8:e019703.

25 Higgins JPT. Chapter 8: Assessing risk of bias in included studies. In: Cochrane handbook for systematic reviews of interventions version 5 The Cochrane Collaboration, 2011. www.handbook.cochrane.org

26 Gagnier JJ, Moher D, Boon H, et al. Investigating clinical heterogeneity in systematic reviews: a methodologic review of guidance in the literature. BMC Med Res Methodol 2012;12:111.

27 Higgins JPT, Thompson SG, Deeks JJ, et al. Measuring inconsistency in meta-analyses. BMJ 2003;327:557-60.

28 Higgins JPT. Chapter 12: Interpreting results and drawing conclusions. In: Cochrane handbook for systematic reviews of interventions version 5. The Cochrane Collaboration, 2011. www. handbook.cochrane.org

29 Fu R, Vandermeer BW, Shamliyan TA, et al. Handling continuous outcomes in quantitative synthesis. In: Methods guide for effectiveness and comparative effectiveness reviews [online]. Agency for Healthcare Research and Quality (US), 2013.

30 Cheung MW-L, Vijayakumar R. A guide to conducting a metaanalysis. Neuropsychol Rev 2016;26:121-8.

31 Popay J, Roberts H, Sowden A, et al. Guidance on the conduct of narrative synthesis in systematic reviews. A product from the ESRC methods programme Version 2006;1:b92.

32 Guyatt GH, Oxman AD, Vist GE, et al. Grade: an emerging consensus on rating quality of evidence and strength of recommendations. $B M J$ 2008;336:924-6.

33 Puhan MA, Schünemann HJ, Murad MH, et al. A grade Working group approach for rating the quality of treatment effect estimates from network meta-analysis. BMJ 2014;349:g5630.

34 Guyatt G, Oxman AD, Akl EA, et al. Grade guidelines: 1. IntroductionGRADE evidence profiles and summary of findings tables. J Clin Epidemiol 2011;64:383-94. 